\title{
Analysis of Clinical and Psychological Characteristics on Mild and Severe COVID-19 Patients
}

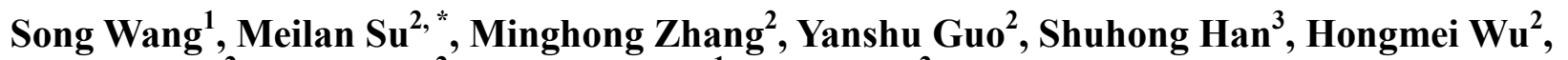 \\ Yunfei Duan², Jing Guan', Zhonghui Zou', Zubin Yin ${ }^{2}$ \\ ${ }^{1}$ Department of Stomach/Thyroid/Vascular Surgery, Chongqing University Three Gorges Hospital, Chongqing, China \\ ${ }^{2}$ Department of Psychosomatic Medicine, Chongqing University Three Gorges Hospital, Chongqing, China \\ ${ }^{3}$ Department of Psychiatry, Chongqing University Three Gorges Hospital, Chongqing, China
}

\section{Email address:}

312069585@qq.com (Meilan Su)

${ }^{*}$ Corresponding author

\section{To cite this article:}

Song Wang, Meilan Su, Minghong Zhang, Yanshu Guo, Shuhong Han, Hongmei Wu, Yunfei Duan, Jing Guan, Zhonghui Zou, Zubin Yin. Analysis of Clinical and Psychological Characteristics on Mild and Severe COVID-19 Patients. Biomedical Sciences.

Vol. 7, No. 1, 2021, pp. 29-35. doi: 10.11648/j.bs.20210701.16

Received: February 22, 2021; Accepted: March 3, 2021; Published: March 12, 2021

\begin{abstract}
Background: Coronavirus disease 2019 (COVID-19) is threatening human physical and mental health. The features of mild and severe cases of COVID-19 may be different, and the impact of psychological intervention is not clear. It is necessary to analyze clinical and psychological characteristics of COVID-19 patients simultaneously. Objective: To evaluate the clinical and psychological differences between mild and severe COVID-19 patients and determine the efficacy of psychological intervention on the patients. Methods: Clinical and psychological data of the patients with COVID-19 were collected. The patients were grouped into mild and severe groups according to their clinical symptoms, and subdivided into psychological intervention group and non-psychological intervention group according to whether they received psychological intervention. The efficiency of psychological interventions in patients with emotional disorder was further explored. Results: 162 participants were included in this study (severe group: 41 patients, mild group: 121 patients), 72 of them received psychological assessment. The demographic, clinical and laboratory characteristics of mild and severe cases were quite different. Most patients in both groups showed mild to moderate anxiety and depression at admission. After psychological intervention for about three weeks, psychological scale scores (HAMA and HAMD score) of patients in the psychological intervention group ( $\mathrm{n}=54$ ) were significantly lower than those in the non-psychological intervention group $(n=18)(P<0.05)$. Conclusions: Early psychological intervention can significantly improve the emotional state of COVID-19 patients. Comprehensive treatments comprising combined clinical and psychological interventions may be effective in the treatment of COVID-19 patients. The physical and mental health of COVID-19 patients requires long-term follow-up.
\end{abstract}

Keywords: COVID-19, Clinical, Depression, Anxiety, Psychological Intervention

\section{Introduction}

Coronavirus disease 2019 (COVID-19), caused by severe acute respiratory syndrome coronavirus 2 (SARS-CoV-2), is spreading around the world and compromising the psychological wellbeing of people. Emotion of fear, panic, stress, and hopelessness spread wantonly [1], aggravating the damage caused by the epidemic. However, the clinical and psychological characteristics of different populations may be different. In terms of treatment, due to the lack of specific drugs for COVID-19, healthcare providers are still relying on supportive therapies, including antiviral and antibacterial agents and glucocorticoids as the main treatment methods [2]. Patients with mild cases usually recover at home or hospital, and no special treatment is required [3]. In severe cases, dyspnea, abnormal coagulation function, liver and kidney damage, acute respiratory distress syndrome (ARDS), multi-organ failure, shock, and even death may occur, they often need to be monitored closely in the intensive care unit (ICU) [4]. For patients with mood 
disorders such as anxiety and depression, psychological intervention may be needed [5]. Whereas, the effectiveness of psychological interventions in patients with COVID-19 remains are unclear. This study aimed to compare the COVID-19 mild and severe groups with regard to the clinical and psychological differences and determine the clinical efficacy of psychological interventions.

\section{Materials and Methods}

\subsection{Research Ethics, Design, and Participants}

The study was approved by the Ethics Committee of the Three Gorges Hospital Affiliated to Chongqing University and informed consent of the patients or their families was obtained. This study included the majority of hospitalized patients admitted to the hospital from January 20 to March 11, 2020, who were diagnosed with COVID-19. The exclusion criteria were pregnancy, death, age $<6$ years, a history of mental illness, and acute exacerbation stage of COPD before onset of COVID-19.

All COVID-19 patients were diagnosed and treated according to the Interim Guidelines of the WHO [6] and the Novel Coronavirus Pneumonia Diagnosis and Treatment Program (NCP-DTP, 7th Edition) of China [7]. According to the NCP-DTP (7th Edition) of China, patients were divided into four types: Mild type: mild clinical symptoms and absence of imaging signs of pneumonia. Ordinary type: fever, respiratory symptoms, and imaging findings of pneumonia. Severe type: with any of the following characteristics: (1) Respiratory distress, (respiratory rate) $\mathrm{RR} \geq 30$ times/min; (2) In the resting state, oxygen saturation $\leq 93 \%$; (3) Partial pressure of arterial oxygen $(\mathrm{PaO} 2)$ /oxygen absorption (FiO2) $\leq 300 \mathrm{mmHg}$. Critically ill type: with one of the following conditions: (1) Respiratory failure that requires mechanical ventilation; (2) Shock; (3) Organ failure that requires ICU support. For the purpose of this study, we categorized the mild and ordinary patients as "mild group," and the severe type and critically ill type patients as "severe group."

\subsection{Clinical and Laboratory Data Acquisition}

Demographic, clinical, and laboratory results, as well as treatment and outcomes, were obtained from the electronic medical record. The specific types of data included demographics, morbidity history, smoking history, potential comorbidities, symptoms, signs, laboratory test results, chest computed tomography (CT) scan results, and main treatment measures. The data were reviewed by a team of trained physicians. The onset date of a patient was defined as the date of symptom onset, and for patients with asymptomatic infection, the onset date was defined as the date of a positive nucleic acid test. Based on the hospitalizations of COVID-19 patients, clinical and laboratory results were compared between the two groups for 25 days.

\subsection{Psychological Assessment and Intervention}

We used the Hamilton Anxiety Scale (HAMA) and
Hamilton Depression Scale (HAMD) to perform the psychological assessments as described in other studies [8,9]. HAMA contains 14 questions, while HAMD contains 17 questions. Each question has five scoring elements: 0 (never), 1 (mild), 2 (moderate), 3 (severe), or 4 (extremely severe). HAMA's total score can be divided into no anxiety (score 0-6), mild anxiety (score 7-13), moderate anxiety (score 14-23), and severe anxiety (score $\geq 24$ ). In contrast, HAMD's total score can be divided into normal (0-7), mild depression (8-17), moderate depression (18-24), and severe depression ( $\geq 25)$.

Psychological scale scoring and psychological intervention were performed by professional psychologists. In this case, the anxiety and depression scales were scored every week, and psychological counseling and interventions were carried out. The psychological interventions were offered face-to-face or through WeChat, telephone, and other methods of communication. The specific psychological interventions included counseling, treatment (such as short-term cognitive behavioral therapy, short-term family support, and short-term focus treatment), and rehabilitation. The clinical indicators and psychological status of the patients were followed up dynamically.

\subsection{Statistical Analysis}

We used SPSS (Social Science Statistical Software Package) version 19.0 software (SPSS 19.0) to perform all statistical analyses. Categorical variables were described as frequency and percentage, and continuous variables were expressed as mean and standard deviation. When the data were normally distributed, the t-test was used to compare the average of continuous variables; otherwise, the Mann-Whitney $U$ test was used. Data from repeated measurements (non-normally distributed) were compared using a generalized linear hybrid model. Chi-square test was used to compare the proportion of taxa, and Fisher's exact test was used when data were limited. Pearson correlation analysis was used to detect the correlation between two sets of data. A value of $\mathrm{P}<0.05$ was considered statistically significant.

\section{Results}

\subsection{General Characteristics}

As of March 11, 2020, a total of 248 patients with COVID-19 were admitted to our hospital: 48 severe patients (including 4 deaths) and 200 mild patients (including 12 asymptomatic patients). The age range of patients was from 7 months and 25 days to 82 years, and one of them had a previous history of mental illness. A total of 162 patients (mild group: 121 patients, severe group: 41 patients) who met inclusion criteria were finally included in the study. Compared to the mild group, the severe group had an older age $(P<0.001)$ and a higher rate of long-term smoking history (smoking time $\geq 10$ years, smoking $\geqq 10$ cigarettes per day) $(P<0.001)$, and were more likely to show cough, fatigue, sputum, and asthma $(P<0.05)$. In terms of comorbidities, patients in the severe group had a higher proportion of cardiovascular disease, type 2 diabetes and 
respiratory diseases than those in the mild group $(P<0.05)$. The basic characteristics of patients in the two groups are shown in Table 1.

\subsection{Clinical and Laboratory Results}

The main clinical examinations, such as the body temperature (Figure 1a) and chest CT, and laboratory indicators, such as the C-reactive protein (CRP) (Figure 1b), procalcitonin (PCT), white blood cell (WBC), total $\mathrm{T}$ lymphocyte (Figure 1c), natural killer cells (NK cells) (Figure

(a) Body temperature $\left({ }^{\circ} \mathrm{C}\right)$

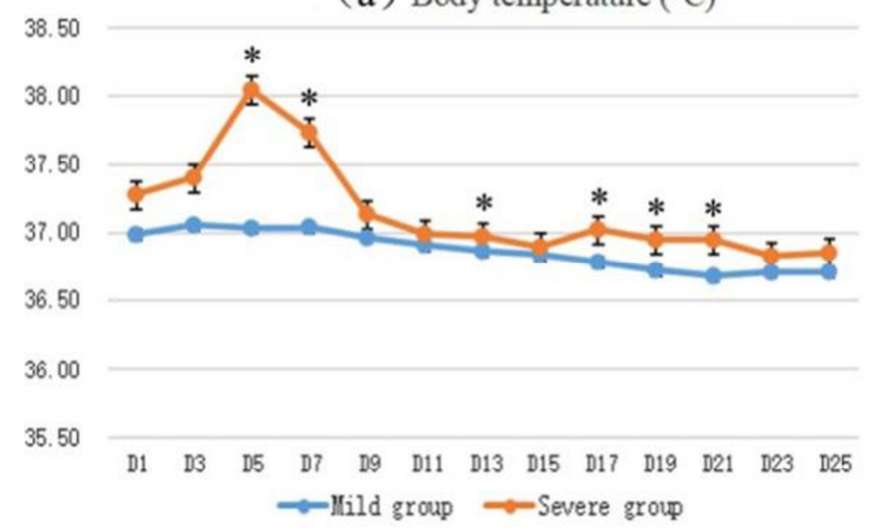

(c) Total T lymphocytes (/u1)

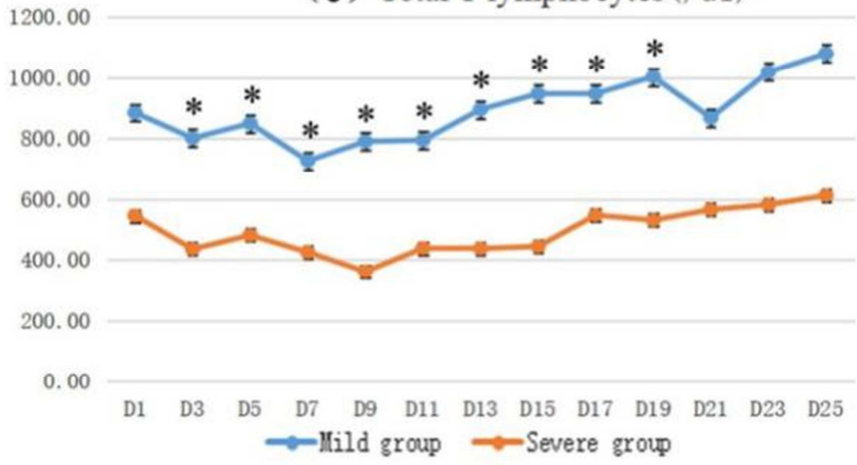

(e) $\operatorname{FDP}(\mathrm{mg} / \mathrm{L})$

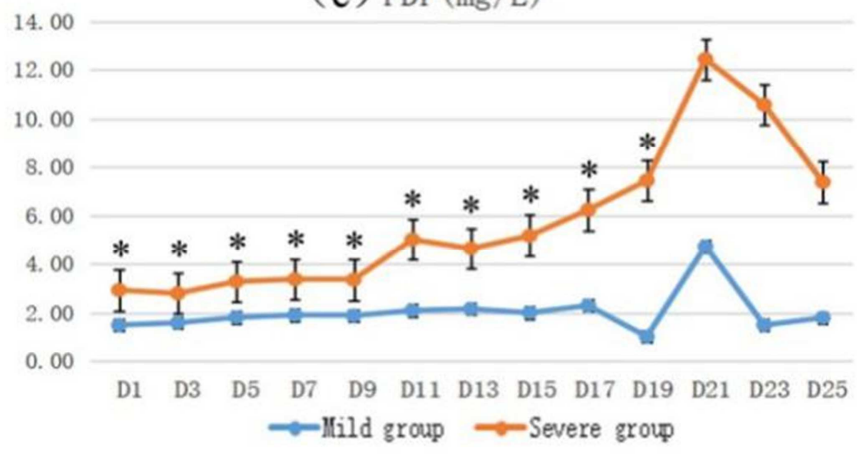

1d), fibrinogen, fibrin degradation products (FDP) (Figure 1e), D-Dimer, albumin, lactate dehydrogenase (LDH), $\alpha$-hydroxybutyrate dehydrogenase $(\alpha-\mathrm{HBDH})$, and aspartate aminotransferase (AST) (Figure 1f), were significantly different between the two groups $(P<0.05)$. However, the $\mathrm{PH}$ value, lactic acid, urea, creatinine, alanine aminotransferase (ALT), cardiac troponin T (cTnT), creatine kinase (CK) and creatine kinase isoenzyme (CK-MB) of the two groups were not significantly different at all time points $(P>0.05)$.

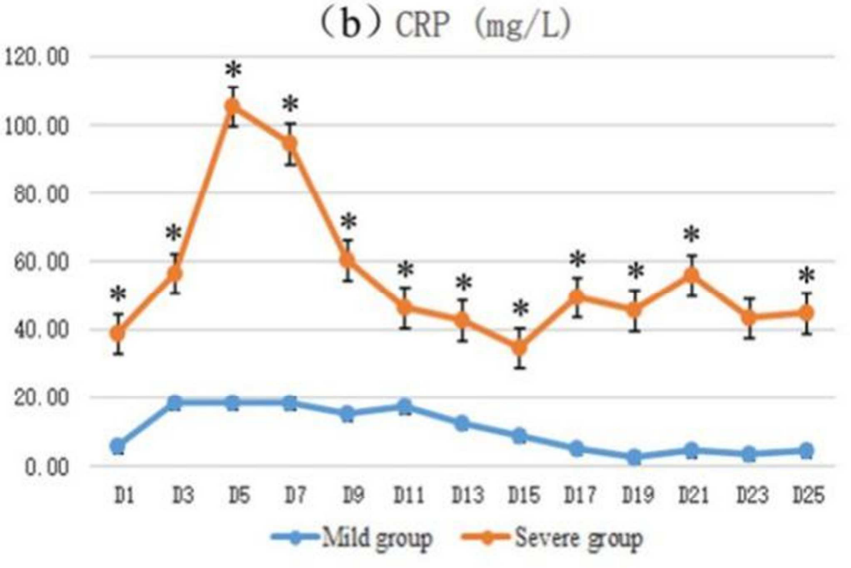

(d) NK cells $(/ \mathrm{ul})$
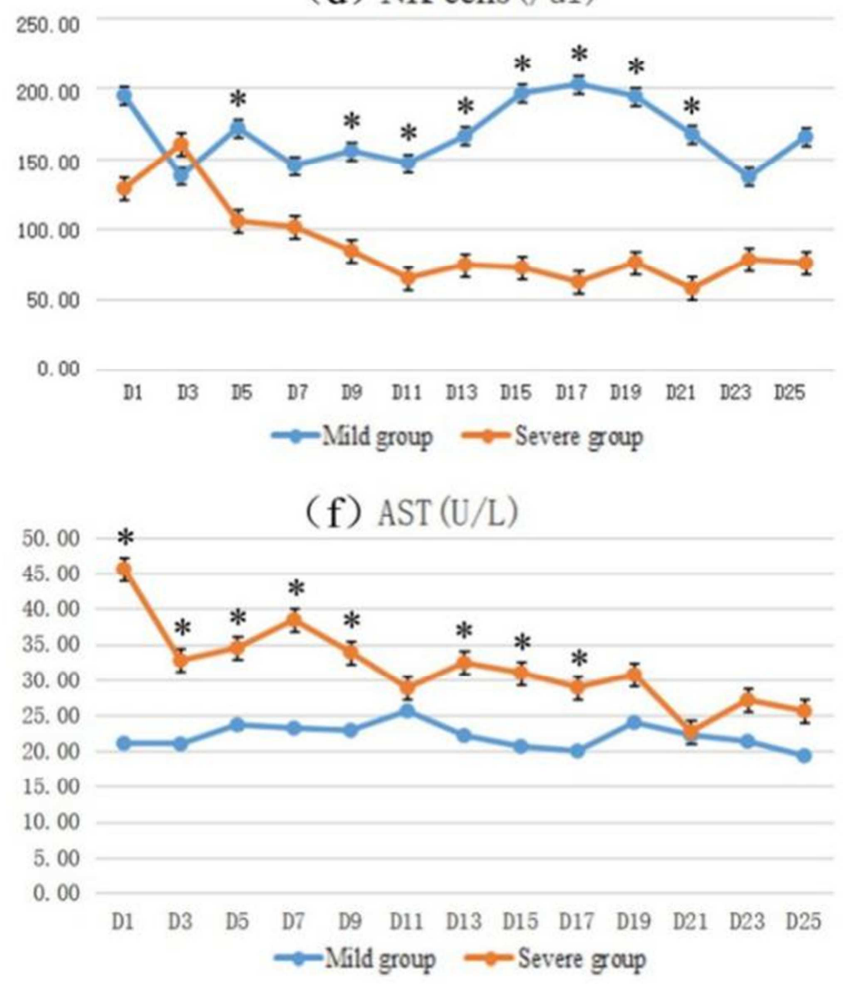

(Note: CRP, C-reactive protein; NK, natural killer; FDP, fibrin degradation products; AST, aspartate aminotransferase. * means $P<0.05$ )

Figure 1. The trends of body temperature (a), CRP (b), total T lymphocyte (c), NK cells (d), FDP (e), and AST (f) in patients with mild and severe COVID-19. 
Table 1. General characteristics of patients in mild and severe groups.

\begin{tabular}{|c|c|c|c|c|}
\hline & \multicolumn{3}{|l|}{ No. $(\%)$} & \multirow{2}{*}{$P$-value } \\
\hline & Total $(n=162)$ & Mild group $(n=121)$ & Severe group $(n=41)$ & \\
\hline Age (mean \pm SD), years & $47.78 \pm 15.45$ & $43.40 \pm 13.28$ & $60.71 \pm 14.24$ & $<0.001$ \\
\hline Gender & & & & 0.936 \\
\hline Male & $90(55.6)$ & $67(55.4)$ & $23(56.1)$ & \\
\hline Female & $72(44.4)$ & $54(44.6)$ & $18(43.9)$ & \\
\hline Long-term smoking history & & & & $<0.001$ \\
\hline Yes & $24(14.8)$ & $11(9.1)$ & $13(31.7)$ & \\
\hline No & $138(85.2)$ & $110(90.9)$ & $28(68.3)$ & \\
\hline \multicolumn{5}{|l|}{ Comorbidities } \\
\hline Cardiovascular disease & $24(14.8)$ & $12(9.9)$ & $12(29.3)$ & 0.003 \\
\hline Diabetes & $17(10.5)$ & $6(5.0)$ & $11(26.8)$ & $<0.001$ \\
\hline Chronic lung disease & $7(4.3)$ & $2(1.7)$ & $5(12.2)$ & 0.015 \\
\hline Chronic liver disease & $4(2.5)$ & $3(2.5)$ & $1(2.4)$ & 1.000 \\
\hline Chronic anemia & $4(2.5)$ & $1(0.8)$ & $3(7.3)$ & 0.083 \\
\hline Malignancy & $2(1.2)$ & $1(0.8)$ & $1(2.4)$ & 0.443 \\
\hline Chronic kidney disease & $2(1.2)$ & $1(0.8)$ & $1(2.4)$ & 0.443 \\
\hline Cerebrovascular disease & $1(0.6)$ & $1(0.8)$ & $0(0)$ & 1.000 \\
\hline \multicolumn{5}{|l|}{ Signs and symptoms } \\
\hline Fever & $114(70.4)$ & $85(70.2)$ & $29(70.7)$ & 0.953 \\
\hline Cough & $118(72.8)$ & $82(67.8)$ & $36(87.8)$ & 0.013 \\
\hline Fatigue & $44(27.2)$ & $24(19.8)$ & $20(48.8)$ & $<0.001$ \\
\hline Expectoration & $28(17.3)$ & $15(12.4)$ & $13(31.7)$ & 0.005 \\
\hline Polypnea & $26(16.0)$ & $11(9.1)$ & $15(36.6)$ & $<0.001$ \\
\hline Muscle ache & $18(11.1)$ & $13(10.7)$ & $5(12.2)$ & 1.000 \\
\hline Headache & $14(8.6)$ & $11(9.1)$ & $3(7.3)$ & 0.978 \\
\hline Sore throat & $12(7.4)$ & $12(9.9)$ & $0(0)$ & 0.080 \\
\hline Anorexia & $13(8.0)$ & $9(7.4)$ & $4(9.8)$ & 0.889 \\
\hline Dizziness & $14(8.6)$ & $8(6.6)$ & $6(14.6)$ & 0.208 \\
\hline Diarrhea & $12(7.4)$ & $7(5.8)$ & $5(12.2)$ & 0.313 \\
\hline Chest pain & $7(4.3)$ & $5(4.1)$ & $2(4.9)$ & 1.000 \\
\hline Stuffy nose, runny nose & $6(3.7)$ & $3(2.5)$ & $3(7.3)$ & 0.348 \\
\hline Nausea and vomiting & $7(4.3)$ & $3(2.5)$ & $4(9.8)$ & 0.125 \\
\hline Abdominal pain & $2(1.2)$ & $0(0)$ & $2(4.9)$ & 0.063 \\
\hline Days from onset to admission, (mean \pm SD) & $6.72 \pm 3.67$ & $6.68 \pm 3.75$ & $6.85 \pm 3.46$ & 0.792 \\
\hline Days from onset to admission, $($ mean $\pm \mathrm{SD})$ & $13.99 \pm 5.32$ & $12.69 \pm 4.32$ & $17.83 \pm 6.15$ & $<0.001$ \\
\hline
\end{tabular}

Note: Long-term smoking history: smoking time $\geq 10$ years, smoking $\geqq 10$ cigarettes per day. SD, standard deviation.

\subsection{Main Interventions, Development, and Outcomes}

Almost all COVID-19 patients received antiviral therapy (such as oseltamivir, abidol, ribavirin, and interferon-a-1b), many patients received antibiotic treatment (e.g., moxifloxacin, piperacillin sodium and tazobactam sodium, cefoperazone, and sulbactam), and glucocorticoids (such as methylprednisolone) treatment. Some patients received protective treatment for the liver, kidney, and stomach. In the severe group, one patient received invasive ventilation (then switched to extracorporeal membrane oxygenation), and one patient received noninvasive ventilation.
In this study, the common complications of COVID-19 (Table 2), such as the acute liver injury, hypoalbuminemia, electrolyte disorder, coagulation dysfunction, ARDS, type I respiratory failure, and acute myocardial injury, occurred more often in the severe group than those in the mild group $(P<0.05)$. Twelve cases in the severe group were developed from mild cases, mainly due to respiratory failure, hepatic insufficiency, electrolyte disturbance, severe infection, acute coronary syndrome, coagulation dysfunction or other aggravated conditions. The average hospital stay in the severe group was significantly longer than in the mild group $(P<0.001)$. 
Table 2. Complications in the mild and severe groups.

\begin{tabular}{|c|c|c|c|c|}
\hline & \multicolumn{3}{|l|}{ No. $(\%)$} & \multirow{2}{*}{$P$-value } \\
\hline & Total $(n=162)$ & Mild group (n=121) & Severe group $(n=41)$ & \\
\hline Acute liver injury & $34(21.0)$ & $19(15.7)$ & $15(36.6)$ & 0.005 \\
\hline Hypoproteinemia & $17(10.5)$ & $2(1.7)$ & $15(36.6)$ & $<0.001$ \\
\hline Electrolyte disturbance & $14(8.6)$ & $2(1.7)$ & $12(29.3)$ & $<0.001$ \\
\hline Coagulation dysfunction & $8(4.9)$ & $0(0)$ & $8(19.5)$ & $<0.001$ \\
\hline ARDS & $8(4.9)$ & $0(0)$ & $8(19.5)$ & $<0.001$ \\
\hline Type I respiratory failure & $5(3.1)$ & $0(0)$ & $5(12.2)$ & 0.001 \\
\hline Acute gastric injury & $3(1.9)$ & $0(0)$ & $3(7.3)$ & 0.015 \\
\hline Acute renal impairment & $2(1.2)$ & $0(0)$ & $2(4.9)$ & 0.063 \\
\hline Sepsis & $2(1.2)$ & $0(0)$ & $2(4.9)$ & 0.063 \\
\hline Shock & $2(1.2)$ & $0(0)$ & $2(4.9)$ & 0.063 \\
\hline
\end{tabular}

Note: ARDS, acute respiratory distress syndrome.

\subsection{Psychological Assessment and Intervention}

Out of the 162 patients included in this study, 54 patients (mild group: 41 cases, severe group: 13 cases) accepted and cooperated during the psychological assessment (HAMA and HAMD score) and psychological intervention for at least three weeks. Another 18 patients (mild group: 14 cases, severe group: 4 cases) accepted psychological evaluation but rejected the psychological intervention. The results of the psychological assessment of the 72 patients (Table 3) showed that almost all patients had mild to moderate anxiety (98.6\%) and depression $(95.8 \%)$ at admission, with no significant difference between the two groups $(P>0.05)$. Meanwhile, no significant correlation was shown between the psychological scale scores (HAMA and HAMD score) and the onset time as well as body temperature at admission $(P>0.05)$.

Table 3. Anxiety and depression of patients in mild and severe groups on admission.

\begin{tabular}{|c|c|c|c|c|}
\hline & \multicolumn{3}{|l|}{ No. (\%) } & \multirow{2}{*}{$P$-value } \\
\hline & Total $(n=72)$ & Mild group $(n=55)$ & Severe group $(n=17)$ & \\
\hline \multicolumn{5}{|l|}{ Anxiety } \\
\hline No & $1(1.4)$ & $1(1.8)$ & $0(0)$ & 1.000 \\
\hline Mild & $26(36.1)$ & $22(40.0)$ & $4(23.5)$ & 0.217 \\
\hline Moderate & $45(62.5)$ & $32(58.2)$ & $13(76.5)$ & 0.173 \\
\hline Severe & $0(0)$ & $0(0)$ & $0(0)$ & - \\
\hline \multicolumn{5}{|c|}{ Depression } \\
\hline Mild & $51(70.8)$ & $37(67.3)$ & $14(82.4)$ & 0.373 \\
\hline Moderate & $18(25.0)$ & $15(27.3)$ & $3(17.6)$ & 0.631 \\
\hline Severe & $0(0)$ & $0(0)$ & $0(0)$ & - \\
\hline
\end{tabular}

Table 4. Comparison of the psychological intervention group and the non-psychological intervention group.

\begin{tabular}{|c|c|c|c|c|}
\hline & \multicolumn{3}{|l|}{ No. $(\%)$} & \multirow[b]{2}{*}{$P$-value } \\
\hline & Total $(n=72)$ & $\begin{array}{l}\text { psychological intervention group } \\
(\mathrm{n}=54)\end{array}$ & $\begin{array}{l}\text { non-psychological intervention } \\
\text { group }(n=18)\end{array}$ & \\
\hline Age (mean \pm SD), years & $44.97 \pm 13.71$ & $44.80 \pm 13.02$ & $45.50 \pm 16.00$ & 0.852 \\
\hline Gender & & & & 0.408 \\
\hline Male & $42(58.3)$ & $33(61.1)$ & $9(50.0)$ & \\
\hline Female & $30(41.7)$ & $21(38.9)$ & $9(50.0)$ & \\
\hline Clinical grouping & & & & 1.000 \\
\hline $\begin{array}{l}\text { Severe group } \\
\text { HAMA scores, }(\text { mean } \pm \mathrm{SD})\end{array}$ & $17(23.6)$ & $13(24.1)$ & $4(22.2)$ & \\
\hline Day 1 & $15.08 \pm 3.73$ & $15.72 \pm 3.59$ & $13.17 \pm 3.57$ & 0.011 \\
\hline $\begin{array}{l}\text { Day } 21 \\
\text { HAMD scores, }(\text { mean } \pm \text { SD) }\end{array}$ & $10.72 \pm 2.93$ & $10.15 \pm 2.49$ & $12.44 \pm 3.52$ & 0.003 \\
\hline Day 1 & $14.60 \pm 4.10$ & $15.46 \pm 3.77$ & $12.00 \pm 4.04$ & 0.001 \\
\hline Day 21 & $9.69 \pm 2.51$ & $9.20 \pm 2.02$ & $11.17 \pm 3.24$ & 0.025 \\
\hline
\end{tabular}

Note: HAMA, Hamilton Anxiety Scale; HAMD, Hamilton Depression Scale; SD, standard deviation.

On admission, the HAMA and HAMD scores of patients in the psychological intervention group were significantly higher than those in the non-psychological intervention group
( $P=0.011$ and 0.001 , respectively). On the 21 th day of admission, these scores of patients in the psychological intervention group were significantly lower than those in the 
non-psychological intervention group $(P=0.003$ and 0.025 , respectively) (Table 4). Compared with admission, the anxiety and depression scores of patients in the psychological intervention group on the 21th day of admission were significantly reduced $(P<0.001)$, while the non-psychological intervention group scores did not change significantly $(P>0.05)$. Besides, no significant difference was observed in the average length of hospital stay between the two groups $(P>0.05)$.

\section{Discussion}

Compared to the mild group, the severe group had an older age, and higher rates of long-term smoking, cardiovascular disease (especially hypertension), and type 2 diabetes. Our findings are consistent with a recent meta-analysis [10], which showed that aged over 65 , smoking patients might face a greater risk of developing into the critical or mortal condition, and the comorbidities such as hypertension, diabetes, cardiovascular disease, and respiratory diseases could also greatly affect the prognosis of the COVID-19. Another meta-analysis [11] showed that cardiovascular disease is an important risk factor for rapid progression and poor prognosis of COVID-19, and it is recommended that COVID-19 patients with cardiovascular disease should take more in-depth medical measures to prevent rapid progression of the disease. A previous study found that all of the above conditions are characterized by increased sympathetic discharge, which may interpret the pathogenesis of these risk factors on COVID-19 patients [12].

Our study has shown that some patients with mild conditions could develop into severe cases due to respiratory failure, liver insufficiency, acute coronary syndrome, or renal insufficiency. Compared with the mild group, some coagulation indicators (such as fibrinogen, FDP and D-Dimer) and liver enzymes (such as LDH, $\alpha$-HBDH and AST) in the severe group were significantly higher, but the immunological indexes (such as total $\mathrm{T}$ lymphocytes and NK cells) were significantly lower. The abnormal coagulation function may be related to the activation of the coagulation system by SARS-CoV-2 [13]. The abnormal liver function may be due to the liver damage caused by SARS-CoV-2 and antiviral drugs [14]. Similarly, the decrease of peripheral blood lymphocytes may be related to the destruction of the immune system caused by SARS-CoV-2 [15]. Hence, the damage of SARS-CoV-2 to the human liver, immune system and coagulation function should be taken seriously. In particular, attention should be paid to the damage of the virus to the liver and immune system, because the frequency of immune cells and liver function are described as useful indicators for prediction of severity and prognosis of COVID-19 patients [15, 16].

During the ongoing COVID-19 pandemic, especially at the beginning of the epidemic, worry, fear, panic, and other adverse emotions are spreading across the population, and many patients are suffering anxiety, depression, and sleep disorders, in addition to suicidal tendencies [17-20]. Early psychological intervention for COVID-19 patients is crucial for their recovery [21]. Our study found that most patients with mild or severe COVID-19 showed mild to moderate anxiety and depression at admission, and no significant correlation was shown between the psychological scale scores (HAMA and HAMD score) and the onset time as well as body temperature at admission. Active psychological intervention could significantly improve the emotional state of patients, although it could not shorten the average hospital stay. In effect, psychological problems in COVID-19 patients will change with disease evolution; therefore, psychological intervention measures should be targeted and adapted as appropriate [5].

There are some limitations to the present study that warrant discussion. Firstly, due to the number of professional psychological medical staff was limited in the region where this study was conducted, and many patients were unwilling to cooperate; only part of the study participants had been subjected to psychological evaluation and intervention for more than three weeks; accordingly, further long-term follow-up research is needed to understand the psychological status of COVID-19 patients. In addition, this study was a retrospective but not a clinical randomized controlled study, and the sample size may be insufficient; thus, additional large-scale studies and randomized controlled study are required.

\section{Conclusion}

In summary, mild and severe COVID-19 patients have different demographic and clinical characteristics, but their psychological characteristics are consistent. Both mild and severe COVID-19 patients are prone to anxiety and depression, and early psychological intervention can significantly improve their emotional state. Comprehensive treatments comprising combined clinical and psychological interventions may be effective in the treatment of COVID-19 patients. The physical and mental health of COVID-19 patients requires long-term follow-up.

\section{Ethics and Funding}

This study was approved by the Ethics Committee of Three Gorges Hospital Affiliated to Chongqing University and funded by the Second Batch of COVID-19 Emergency Prevention and Control Science and Technology Project in Wanzhou District, Chongqing (Project No.: WZSTC-2020016).

\section{Author Contributions}

Wang S and Su ML contributed to the study design, data collection, statistics and paper writing; Han SH contributed to psychological tests and intervention; Guo YS, Wu HM, Duan YF, Guan J, Zou ZH, and Yin ZB contributed to data collection. All authors have approved the final version to be published. 


\section{Conflict of Interests}

All the authors do not have any possible conflicts of interest.

\section{Acknowledgements}

We are grateful for the clinical cases provided by Chongqing University Three Gorges Hospital and for the language editing of language editing company Editage.

\section{References}

[1] Ozamiz-Etxebarria N, Dosil-Santamaria M, PicazaGorrochategui M, et al. Stress, anxiety, and depression levels in the initial stage of the COVID-19 outbreak in a population sample in the northern Spain. Cad Saude Publica. 2020, 36 (4): $\mathrm{e} 00054020$.

[2] Wang D, Hu B, Hu C, et al. Clinical characteristics of 138 hospitalized patients with 2019 Novel Coronavirus-Infected pneumonia in Wuhan, China. JAMA. 2020, 323 (14): 1406-7.

[3] Gandhi RT, Lynch JB, Del Rio C. Mild or Moderate Covid-19. N Engl J Med. 2020, 383 (18): 1757-1766.

[4] Huang C, Wang Y, Li X, et al. Clinical features of patients infected with 2019 novel coronavirus in Wuhan, China. Lancet. 2020, 395 (10223): 497-506.

[5] Duan L, Zhu G. Psychological interventions for people affected by the COVID-19 epidemic. Lancet Psychiatry. 2020, 7 (4): 300-302.

[6] World Health Organization. Clinical management of severe acute respiratory infection when novel coronavirus $(\mathrm{nCoV})$ infection is suspected: interim guidance, [Accessed 31 January 2020]. https://www.who.int/publications/i/item/clinical-managementof-covid-19.

[7] The General Office of the National Health and Health Commission of the People's Republic of China, the Office of the State Administration of Traditional Chinese Medicine. The Novel Coronavirus Pneumonia Diagnosis and Treatment Program (7th Edition) of China. Chin Med. 2020, 15 (6): 801-5.

[8] Zimmerman M, Thompson JS, Diehl JM, et al. Is the DSM-5 Anxious Distress Specifier Interview a valid measure of anxiety in patients with generalized anxiety disorder: A comparison to the Hamilton Anxiety Scale. Psychiatry Res. 2020, 286: 112859 .
[9] Moazen-Zadeh E, Bayanati S, Ziafat K, et al. Vortioxetine as adjunctive therapy to risperidone for treatment of patients with chronic schizophrenia: A randomized, double-blind, placebo-controlled clinical trial. J Psychopharmacol. 2020, 34 (5): 506-13.

[10] Zheng Z, Peng F, Xu B, et al. Risk factors of critical \& mortal COVID-19 cases: A systematic literature review and meta-analysis. $J$ Infect. 2020, 81 (2): 16-25.

[11] Kunutsor SK, Laukkanen JA. Cardiovascular complications in COVID-19: A systematic review and meta-analysis. $J$ Infect. 2020, 81 (2): e139-141.

[12] Porzionato A, Emmi A, Barbon S, et al. Sympathetic activation a potential link between comorbidities and COVID-19. FEBS J. 2020, 287 (17): 3681-3688.

[13] Lemke G, Silverman GJ. Blood clots and TAM receptor signaling in COVID-19 pathogenesis. Nat Rev Immunol. 2020, 20 (7): 395-396.

[14] Cai Q, Huang D, Yu H, et al. COVID-19: Abnormal liver function tests. $J$ Hepatol, 2020, 73 (3): 566-574.

[15] Taghiloo S, Aliyali M, Abedi S, et al. Apoptosis and immunophenotyping of peripheral blood lymphocytes in Iranian COVID-19 patients: Clinical and laboratory characteristics. J Med Virol. 2020 Sep 10.

[16] Piano S, Dalbeni A, Vettore E, et al. Abnormal liver function tests predict transfer to intensive care unit and death in COVID-19. Liver Int. 2020, 40 (10): 2394-2406.

[17] Sher L. COVID-19, anxiety, sleep disturbances, and suicide. Sleep Med. 2020, 70: 124.

[18] Solomou I, Constantinidou F. Prevalence and Predictors of Anxiety and Depression Symptoms during the COVID-19 Pandemic and Compliance with Precautionary Measures: Age and Sex Matter. Int J Environ Res Public Health. 2020, 17 (14): 4924.

[19] Rogers JP, Chesney E, Oliver D, et al. Psychiatric and neuropsychiatric presentations associated with severe coronavirus infections: a systematic review and meta-analysis with comparison to the COVID-19 pandemic. Lancet Psychiatry. 2020, (7): 611-27.

[20] Kawohl W, Nordt C. COVID-19, unemployment, and suicide. Lancet Psychiatry. 2020, 7 (5): 389-90.

[21] Gonzalez-Sanguino C, Ausin B, AngelCastellanos M, et al. Mental health consequences during the initial stage of the 2020 Coronavirus pandemic (COVID-19) in Spain. Brain Behav Immun. 2020, 87: 172-176. 